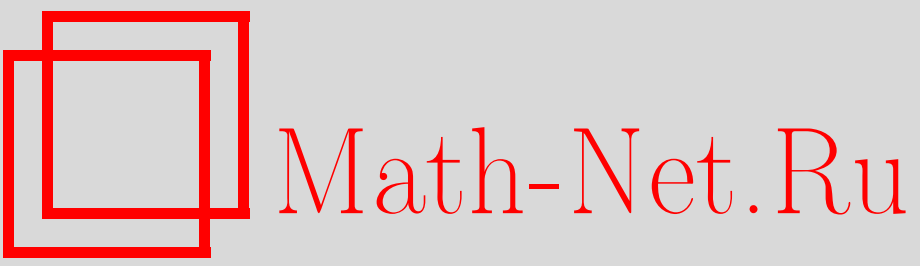

Д. А. Чечин, А. Д. Баев, С. А. Шабров, Об одной граничной задаче с разрывными решениями и сильной нелинейностью, Итоги науки и техн. Сер. Соврем. мат. и ее прил. Темат. обз., 2021, том 193, 153-157

DOI: https://doi.org/10.36535/0233-6723-2021-193-153-157

Использование Общероссийского математического портала Math-Net.Ru подразумевает, что вы прочитали и согласны с пользовательским соглашением

http://www.mathnet.ru/rus/agreement

Параметры загрузки:

IP : 54.174 .149 .18

26 апреля 2023 г., $18: 17: 28$ 


\title{
ОБ ОДНОЙ ГРАНИЧНОЙ ЗАДАЧЕ \\ С РАЗРЫВНЫМИ РЕШЕНИЯМИ \\ И СИЛЬНОЙ НЕЛИНЕЙНОСТЬЮ
}

(c) 2021 г. Д. А. ЧЕЧИН, А. Д. БАЕВ, С. А. ШАБРОВ

\begin{abstract}
АннотАция. В работе получены достаточные условия существования решения краевой задачи второго порядка с разрывными решениями и сильной нелинейностью. При анализе решений краевой задачи мы используем поточечный подход, предложенный Ю. В. Покорным и показавший свою эффективность при изучении задач второго порядка с негладкими решениями. На основе оценок функции Грина граничной задачи, полученных ранее другими авторами, удалось показать, что оператор, обращающий изучаемую нелинейную задачу, представимый в виде суперпозиции вполне непрерывного и непрерывного операторов, действует из конуса неотрицательных непрерывных функций в более узкое множество. Последнее и позволяет доказать существование решения у нелинейной краевой задачи с привлечением теории пространств с конусом.
\end{abstract}

Ключевые слова: краевая задача, негладкое решение, сильная нелинейность, разрешимость.

\section{ON A BOUNDARY-VALUE PROBLEM WITH DISCONTINUOUS SOLUTIONS AND STRONG NONLINEARITY}

\author{
(c) 2021 D. A. CHECHIN, A. D. BAEV, S. A. SHABROV
}

\begin{abstract}
In this work, sufficient conditions for the existence of a solution to a second-order boundary-value problem with discontinuous solutions and strong nonlinearity are obtained. For the analysis of solutions to the boundary-value problem, we apply the pointwise approach proposed by Yu. V. Pokornyi and which has shown its effectiveness in studying second-order problems with nonsmooth solutions. Based on estimates of the Green function of the boundary-value problem obtained earlier by other authors, we show that the operator, which inverts the nonlinear problem considered, can be represented as the composition of a completely continuous operator and a continuous operator; this operator acts from the cone of nonnegative continuous functions into a narrower set. This fact allows one to prove the existence of a solution to a nonlinear boundary-value problem by using the theory of spaces with a cone.
\end{abstract}

Keywords and phrases: boundary-value problem, nonsmooth solution, strong nonlinearity, solvability.

AMS Subject Classification: 34A36, 34A34

1. Введение. В последние десятилетия активно ведутся исследования дифференциальных операторов Штурма-Лиувилля (Шредингера) для случаев разнообразных импульсных возмущений (сингулярных потенциалов). Можно выделить публикации А. А. Шкаликова, А. М. Савчука, Б. С. Митягина, В. А. Михайлеца (см. $[5-8,15,16])$ и др., в которых методами теории обобщенных

Работа выполнена при поддержке Российского научного фонда (проект № 19-11-00197). 
функций решаются задачи, касающиеся структуры спектра, его асимптотики, спектральной полноты, разнообразных свойств непрерывного спектра, структуры сингулярных компонент спектра (спектральных лакун, зон неустойчивости) и проч.

Следует отметить, что поточечный анализ решения у математической модели очень важен для приложений. Наличие особенностей у изучаемой системы (как внутренних, так и внешних), как правило, приводит не только к потере гладкости решения дифференциальной модели, но и появлению разрывов у решения. Последнее, как следствие, закрывает возможность использования классических производных как при моделировании, так и при анализе. Применение теории обобщенных функций (по Соболеву-Шварцу, Коломбо и др.) ситуацию не спасает: удается доказать лишь наличие слабого решения. При этом приходится преодолевать ряд трудноразрешимых проблем (например, умножения разрывной функции на обобщенную).

Подход к поточечному анализу решений уравнения

$$
-\left(p u^{\prime}\right)^{\prime}+q u=f
$$

в случае особенностей типа функции Дирака в потенциале $q$, когда $q$ может считаться обобщенной производной от функции ограниченной вариации $Q$, был разработан Ю. В. Покорным в $[9,10]$, где в развитие идей В. Феллера и М. Г. Крейна (см. комментарии в [1]), уравнение с обобщенными коэффициентами и правой частью

$$
-\left(p u^{\prime}\right)^{\prime}+Q^{\prime} u=F^{\prime}
$$

заменялось поточечно задаваемым уравнением

$$
-\left(p u^{\prime}\right)_{\sigma}^{\prime}+u Q_{\sigma}^{\prime}=F_{\sigma}^{\prime} .
$$

Дальнейшее развитие этот подход для случая непрерывных решений получил в работах $[11,12]$.

Для случая разрывных решений предыдущее уравнение имеет вид

$$
-\left(p u_{\mu}^{\prime}\right)_{[\sigma]}^{\prime}+u Q_{[\sigma]}^{\prime}=F_{[\sigma]}^{\prime} .
$$

Здесь $p, Q, F$ - функции ограниченной вариации на отрезке $[0, \ell]$; решения $u(x)$ принадлежат классу $\mu$-абсолютно непрерывных функций на $[0, \ell], \mu$-производные которых имеют ограниченную вариацию на $[0, \ell]$; внутреннее дифференцирование ведется по $\mu$-мере, порождаемой строго возрастающей на $[0, \ell]$ функцией $\mu(x)$, соизмеримой с наблюдаемым процессом. Строго возрастающую функцию $\sigma(x)$ можно подобрать таким образом, что она будет нести в себе все особенности рассматриваемой задачи. Точнее, $\sigma(x)$ будет являться разрывной лишь в точках разрыва $\mu, p, Q$, $F$, так что $[\sigma]$-мера этих точек оказывается отличной от нуля. При этом $[\sigma]$-мера точек разрыва $u(x)$ «расщепляется» в силу того, что функция $\sigma(x)$ в этих точках имеет два скачка (левый и правый), равных $\Delta^{-} \sigma(\xi)=\sigma(\xi)-\sigma(\xi-0)$ и $\Delta^{+} \sigma(\xi)=\sigma(\xi+0)-\sigma(\xi)$ соответственно. Чтобы подчеркнуть, что речь идет о «расщепленной» мере, мы заключаем порождающую меру функцию в квадратные скобки. Отметим, что для восстановления функции по ее $[\sigma]$-производной необходимо использовать $\pi$-интеграл, введенный Ю. В. Покорным в [10].

В настоящей работе мы применяем подход Ю. В. Покорного к одной граничной задаче с сильной нелинейностью.

2. Основной результат. Рассмотрим на $[0 ; \ell]$ нелинейную математическую модель

$$
\left\{\begin{array}{l}
L u \equiv-\left(p u_{\mu}^{\prime}\right)_{[\sigma]}^{\prime}+u Q_{[\sigma]}^{\prime}=f(x, u), \\
u(0)=u(\ell)=0,
\end{array}\right.
$$

возникающую, например, при моделировании деформаций разрывной струны (цепочки из струн, скрепленных между собой пружинами), натянутой вдоль отрезка $[0, \ell]$, и подпертой (не более чем в счетном количестве точек), как обычными пружинами, деформации которых подчиняются закону Гука, так и пружинами с разными витками, деформации которых закону Гука не подчиняются и задаются некоторой функцией. При этом к отдельным точкам струны могут быть приложены нелинейные импульсные внешние воздействия.

В уравнении из (1) внутренняя производная понимается как производная по обычной мере, внешняя - как производная по «расщепленной» мере, понимаемая в смысле Ю. В. Покорного, 
т. е. обращаемая интегрированием с помощью $\pi$-интеграла. Последнее означает, что функция $g(x)$ называется $[\sigma]$-производной от функции $G(x)$, если

$$
G(x)-G(0)=\int_{0}^{x} g(s) d[\sigma] .
$$

Таким образом, во всякой точке $\xi$ разрыва функции $\sigma(x)$ у функции $g(x)$ возникает два собственных значения, вообще говоря, отличных от предельных, определяемых равенствами

$$
g\left(\xi^{1}\right)=G_{[\sigma]}^{\prime}\left(\xi^{1}\right)=\frac{\Delta^{-} G(\xi)}{\Delta^{-} \sigma(\xi)}, \quad g\left(\xi^{2}\right)=G_{[\sigma]}^{\prime}\left(\xi^{2}\right)=\frac{\Delta^{+} G(\xi)}{\Delta^{+} \sigma(\xi)} .
$$

Уравнение (1) рассматривается на специальном расширении $\overline{[0 ; \ell]}_{[\sigma]}$ отрезка $[0 ; \ell]$, которое строится следующим образом. Обозначим через $S(\mu)$ множество точек разрыва функции $\mu(x)$. На множестве $J_{\mu}=[0 ; \ell] \backslash S(\mu)$ введем метрику $\rho(x ; y)=|\mu(x)-\mu(y)|$. Метрическое пространство $J_{\mu}$ является неполным. Обозначим через $\overline{[0 ; \ell]}_{\mu}$ его стандартное пополнение. Множество $\overline{[0 ; \ell]}_{\mu}$ вместо каждой точки $\xi$ разрыва функции $\mu(x)$ содержит элементы $\left\{\xi^{1} ; \xi^{2}\right\}$, появившиеся при пополнении. При этом $x<\xi^{1}<\xi^{2}<y$ в смысле естественной упорядоченности элементов, если $x<\xi<y$. Определим $u\left(\xi^{1}\right)=u(\xi-0), u\left(\xi^{2}\right)=u(\xi+0)$. Пусть $S$ - множество точек разрыва функции $\sigma(x)$, не являющихся точками разрыва $\mu(x)$. Рассмотрим множество $\overline{[0 ; \ell}_{\mu} \backslash S$, пополним его по метрике $\rho(x ; y)=|\sigma(x)-\sigma(y)|$ и добавим к полученному пополнению элементы из $S$. Обозначим данное множество через $\overline{[0, \ell]}_{[\sigma]}$. Обозначим ${\overline{[0, \ell]_{S}}}_{S}=\overline{[0, \ell]}_{[\sigma]} \bigcup S(\mu)$.

Таким образом, в точках $\xi^{1}$ и $\xi^{2}$ уравнение (1) имеет вид

$$
\begin{aligned}
& -\Delta^{-}\left(p u_{\mu}^{\prime}\right)(\xi)+u(\xi-0) \Delta^{-} Q(\xi)=f\left(\xi^{1}, u(\xi-0)\right) \Delta^{-} \sigma(\xi), \\
& -\Delta^{+}\left(p u_{\mu}^{\prime}\right)(\xi)+u(\xi+0) \Delta^{+} Q(\xi)=f\left(\xi^{2}, u(\xi+0)\right) \Delta^{+} \sigma(\xi) .
\end{aligned}
$$

В точках $s$ разрыва функции $\sigma(x)$, в которых $\mu(x)$ является непрерывной, уравнение (1) имеет вид

$$
-\Delta\left(p u_{\mu}^{\prime}\right)(s)+u(s) \Delta Q(s)=f(s, u(s)) \Delta \sigma(s),
$$

где $\Delta v(s)=v(s+0)-v(s-0)$.

Решение (1) мы будем искать в классе $E \mu$-абсолютно непрерывных на $\overline{[0 ; \ell]}_{\mu}$ функций, первая производная которых $[\sigma]$-абсолютно непрерывна на $\overline{[0 ; \ell}_{S}$. Относительно коэффициентов $p(x)$, $Q(x)$ и функции $f(x, u)$ мы делаем следующие предположения:

(I) $p(x)$ и $Q(x)-[\sigma]$-абсолютно непрерывны на $\overline{[0 ; \ell]}_{S}$;

(II) функция $p(x)$ положительна и отделена от нуля;

(III) функция $Q(x)$ не убывает на $[0 ; \ell]$;

(IV) $f(x, u)$ удовлетворяет условию Каратеодери, т. е.

(а) при каждом фиксированном $u$ функция $f(x, u)$ является $[\sigma]$-измеримой;

(b) при всех $x \in \overline{[0 ; \ell]}_{\mu} f(x, u)$ - непрерывна по $u$;

(c) существует $[\sigma]$-суммируемая с некоторой степенью $p \in[1, \infty)$ функция $m(x)$ такая, что $|f(x, u)| \leqslant m(x)$ для почти всех $x$ (в смысле $[\sigma]$-меры) и $u$.

Нетрудно видеть, при выполнении последних условий оператор суперпозиции $[\mathbf{F} u](x)=f(x, u(x))$ непрерывно действует из $C_{\mu}$ - пространства $\mu$-непрерывных на $\overline{[0 ; \ell]}_{\mu}$ функций в $L_{r,[\sigma]}-[\sigma]$-суммируемых с некоторой степенью $r$ функций.

При выполнении условий (I)-(III) достаточно легко доказать разрешимость в классе $E$ линейной задачи (когда в правой части в (1) стоит $f(x)-[\sigma]$-суммируемая функция); показать существование и единственность функции влияния $H(x, s)$ линейной граничной задачи $L u=f$, $u(0)=u(\ell)=0$. Ключевым моментом для наших результатов является следующая

Теорема 1. Пусть выполнены условия (I)-(III). Тогда существуют такое положительное число $\kappa$ и такие $[\sigma]$-суммируемые почти всюду положительные функиии $v_{1}(s), v_{2}(s)$, что для 
всех $x, s, \tau$, принадлежсащих $\overline{[0 ; \ell]}_{\mu}$, справедливъ неравенства

$$
\begin{gathered}
H(x, s) \geqslant \kappa u_{0}(x) H(\tau, s), \\
u_{0}(x) v_{1}(s) \leqslant H(x, s) \leqslant u_{0}(x) v_{2}(s),
\end{gathered}
$$

где $u_{0}(x)=(\mu(x)-\mu(0))(\mu(\ell)-\mu(x))$.

Основным результатом работы является теорема

Теорема 2. Пусть выполнены следующие условия:

(1) $f(x, 0) \equiv 0$;

(2) однородное уравнение $L u=0$ не осииллирует на $\overline{[0 ; \ell]}$ [б]

(3) $f(x, u) \geqslant 0$ для всех $x \in[0 ; \ell] u \quad u \geqslant 0$;

(4) оператор суперпозиции, порожденный функиией $f(x, u)$, непрерывно действует из $C_{\mu}[0 ; \ell]$ в $L_{p,[\sigma]} \overline{[0 ; \ell]}_{[\sigma]}$ при некотором $p \in(1 ;+\infty]$

(5) при некоторых $0<r<R<\infty$ краевая задача $L u=\lambda f(x, u), u(0)=0, u(1)=0$, при любъх $\lambda \in(0 ; 1)$ не имеет решений, удовлетворяющих неравенствам

$$
\widetilde{u}_{0}(x) \cdot \max _{0 \leqslant x \leqslant 1}|u(x)| \leqslant u(x) \leqslant r
$$

где $\widetilde{u}_{0}(x)=M \cdot u_{0}(x)$ при некотором $M>0$, и для некоторой неотрицательной нетривиальной бункиии $h(x) \in L_{1,[\sigma]} \overline{[0 ; 1]}_{[\sigma]} u$ для любого $\lambda>0$ граничная задача $L u=\lambda f(x, u)+\lambda h$, $u(0)=0, u(1)=0$, не имеет решений, удовлетворяющих неравенству $u(x) \geqslant R \tilde{u}_{0}(x)$.

Тогда задача

$$
L u=f(x, u), \quad u(0)=0, \quad u(1)=0
$$

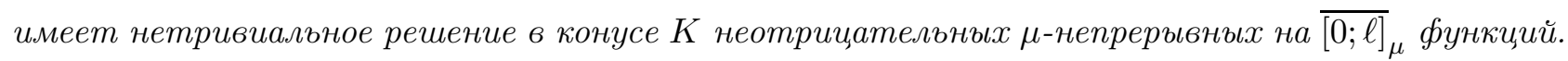

Доказательство. С помощью оператора $A$ на $K \backslash\{\Theta\}$ (здесь $\Theta$ - нулевой элемент в множестве непрерывных на $[0 ; \ell]$ функций) введем оператор

$$
B u=\|u\|_{C}^{2} A\left(\frac{u}{\|u\|_{C}^{2}}\right) .
$$

Если оператор $B$ имеет неподвижную точку $u^{*}$, то элемент $v^{*}=u^{*} /\|u\|_{C}^{2}$ дает неподвижную точку оператора $A$. Поэтому достаточно показать наличие в $K$ у оператора $B$ неподвижной точки.

Оператор $B$ переводит $K \backslash\{\Theta\}$ в $K\left(\tilde{u}_{0}\right)$, причем $B$ вполне непрерывен в $K$ вне шара любого радиуса. Нетрудно видеть, что для оператора $B$ на множестве элементов $K\left(\tilde{u}_{0}\right)$ с большой нормой не может выполняться $\lambda B u=u$ при $\lambda \in(0,1)$, и на элементах малой нормы из $K\left(\tilde{u_{0}}\right)$ при любом $\lambda>0$ не может выполняться $u=B u+\lambda h_{0}$, где

$$
h_{0}(x)=\int_{0}^{1} G(x, s) h(s) d \sigma(s) .
$$

Поэтому оператор $B$ имеет в $K\left(\tilde{u}_{0}\right)$ неподвижную точку. Теорема доказана.

Отметим, что доказательство теоремы сохраняет силу, если оператор $A$ вполне непрерывен вне любого шара положительного радиуса.

\section{СПИСОК ЛИТЕРАТУРЫ}

1. Аткинсон $\Phi$. Дискретные и непрерывные граничные задачи. - М.: Мир, 1968.

2. Баев А. Д., Шабров С. А., Голованева Ф. В., Меач Мон. Функция влияния дифференциальной модели четвертого порядка// Вестн. Воронеж. ин-та ГПС МЧС. - 2014. - 3, № 12. - С. 65-73.

3. Давыдова М. Б., Шабров С. А. О числе решений нелинейной краевой задачи с интегралом Стилтьеса// Изв. Саратов. ун-та. Нов. сер. Сер. Мат. Мех. Информ. - 2011. - 11, № 4. - С. 13-17.

4. Давыдова М. Б., Шабров С. А. О нелинейных теоремах сравнения для дифференциальных уравнений второго порядка с производными Радона-Никодима// Вестн. Воронеж. гос. ун-та. Сер. Физ. Мат. 2013. - № 1. - C. 155-160. 
5. Иванов A. C., Савчук A. М. След порядка минус один для струны с сингулярными весом// Мат. заметки. - 2017. - 102, № 2. - С. 197--215.

6. Конечная Н. Н., Мирзоев К. А., Шкаликов А. А. Об асимптотике решений двучленных дифференциальных уравнений с сингулярными коэффициентами// Мат. заметки. - 2018. - 104, № 2. - С. $231-242$.

7. Митягин Б. С., Джаков П. Б. Зоны неустойчивости одномерных периодических операторов Шредингера и Дирака// Усп. мат. наук. - 2006. - 61, № 4 (370). - С. 77-182.

8. Михайлеи, B. А. Структура непрерывного спектра одномерного оператора Шредингера с точечными взаимодействиями // Функц. анал. прилож. - 1996. - 30, № 2. - С. 90-93.

9. Покорныц Ю. В. Интеграл Стилтьеса и производные по мере в обыкновенных дифференциальных уравнениях // Докл. РАН. - 1999. - 364, № 2. - С. 167-169.

10. Покорный Ю. В. О дифференциалах Стилтьеса в обобщенной задаче Штурма-Лиувилля// Докл. РАН. - 2002. - 383, № 5. - C. 1-4.

11. Покорный Ю. В., Бахтина Ж. И., Зверева М. Б., Шабров С. А. Осцилляционный метод Штурма в спектральных задач. - М.: Физматлит, 2009.

12. Покорный Ю. В., Зверева М. Б., Шабров С. А. Осцилляционная теория Штурма-Лиувилля для импульсных задач// Усп. мат. наук. - 2008. - 63, № 1 (379). - С. 111-154.

13. Покорныц Ю. В., Зверева М. Б., Ищенко А. С., Шабров С. А. О нерегулярном расширении осцилляционной теории спектральной задачи Штурма-Лиувилля// Мат. заметки. - 2007. - 82, № 4. C. $578-582$.

14. Покорный Ю. В., Пенкин О. М., Прядиев В. Л., Боровских А.В., Лазарев К. П., Шабров С. А. Дифференциальные уравнения на геометрических графах. - М.: Физматлит, 2004.

15. Савчук A. М. О базисности системы собственных и присоединенных функций одномерного оператора Дирака// Изв. РАН. Сер. мат. - 2018. - 82, № 2. - С. 113-139.

16. Савчук A. М., Шкаликов A. А. Обратные задачи для оператора Штурма-Лиувилля с потенциалами из пространств Соболева. Равномерная устойчивость // Функц. анал. прилож. - 2010. - 44, № 4. C. $34-53$.

17. Шабров C. А. Об одной математической модели малых деформаций стержневой системы с внутренними особенностями // Вестн. Воронеж. гос. ун-та. Сер. Физ. Мат. - 2013. - № 1. - С. 232-250.

18. Шабров $C$. A. Об оценках функции влияния одной математической модели четвертого порядка// Вестн. Воронеж. гос. ун-та. Сер. Физ. Мат. - 2015. - 2. - С. 168-179.

19. Pokornyi Yu. V., Shabrov S. A. Toward a Sturm-Liouville theory for an equation with generalized coefficients// J. Math. Sci. — 2004. - 119, № 6. - P. 769-787.

20. Pokornyi Yu. V., Zvereva M. B., Shabrov S. A. On extension of the Sturm-Liouville oscillation theory to problems with pulse parameters// Ukrain. Math. J. - 2008. - 60, № 1. - P. 108-113.

Чечин Дмитрий Александрович

Воронежский государственный университет

E-mail: alexsandrbaev@mail.ru

Баев Александр Дмитриевич

Воронежский государственный университет

E-mail: alexsandrbaev@mail.ru

Шабров Сергей Александрович

Воронежский государственный университет

E-mail: shaspoteha@mail.ru 